\title{
Ernährung in der Schwangerschaft - Handlungsempfehlungen des Netzwerks "Gesund ins Leben - Netzwerk Junge Familie“
}

\author{
B. Koletzko, C.-P. Bauer, P. Bung, M. Cremer, M. Flothkötter, C. Hellmers, \\ M. Kersting, M. Krawinkel, H. Przyrembel, R. Rasenack, \\ T. Schäfer, K. Vetter, U. Wahn, A. Weißenborn, A. Wöckel
}

\section{Einleitung}

Eine gesunde Lebensweise in der Schwangerschaft ist wichtig für Mutter und Kind. Eine ausgewogene Ernährung und regelmäßige körperliche Bewegung vor und während der Schwangerschaft wirken sich nicht nur kurzfristig aus, sondern haben auch langfristig positive Auswirkungen auf Gesundheit und Wohlbefinden.

Für werdende Eltern ist die Zeit der Schwangerschaft intensiv, spannend und herausfordernd zugleich. Sie möchten das Richtige für die gesunde Entwicklung ihres Kindes tun und dem Nachwuchs einen guten Start ins Leben ermöglichen. Dafür müssen sie zahlreiche Entscheidungen treffen. Besonders die Themen Ernährung in der Schwangerschaft und auch Allergievorbeugung (für das Kind) liegen vielen werdenden Eltern am Herzen. Im Alltag werden sie dabei jedoch häufig mit unterschiedlichen Aussagen konfrontiert. Deshalb hat sich das Netzwerk „Gesund ins Leben - Netzwerk Junge Familie“ zum Ziel gesetzt, harmonisierte Handlungsempfehlungen als Basis für Kommunikationsmaßnahmen und Medien des Netzwerks zu entwickeln.

\section{Material und Methode}

Dazu erfolgte durch den wissenschaftlichen Beirat des Netzwerks zwischen 2009 und 2011 eine Sichtung und Be- wertung einschlägiger Publikationen, Metaanalysen und Leitlinien sowie von Empfehlungen und Referenzwerten zur Nährstoffzufuhr von Fachorganisationen und Institutionen, die Aussagen zu Ernährung und Gesundheit von Schwangeren und/oder zur Allergievorbeugung treffen (Berufsverband der Frauenärzte, Bundesinstitut für Risikobewertung, Bundeszentrale für gesundheitliche Aufklärung, Cochrane Library, Deutsche Gesellschaft für Ernährung, Deutsche Gesellschaft für Gynäkologie und Geburtshilfe, Deutsche Gesellschaft für Kinder- und Jugendmedizin, Deutscher Hebammenverband, Europäische Kommission, Europäische Behörde für Lebensmittelsicherheit, Forschungsinstitut für Kinderernährung, Institute of Medicine [USA], National Institute for Health and Clinical Excellence [UK], Nationale Stillkommission am Bundesinstitut für Risikobewertung, Weltgesundheitsorganisation).

Die Autoren haben sich bei der Entwicklung der Empfehlungen vor allem auf vorhandene Leitlinien, Metaanalysen und systematische Übersichten gestützt. Im Rahmen des Netzwerks wurde keine eigene systematische Literatursuche durchgeführt. Die im Konsens formulierten Kernaussagen entsprechen damit dem Evidenzniveau einer Expertenempfehlung. Sie sollten in regelmäßigen Abständen, spätestens nach 5 Jahren, aktualisiert werden, um ihre Gültigkeit zu behalten. Die Autoren halten hierbei eine systematische Literaturrecherche für wünschenswert.
Die Handlungsempfehlungen wurden im Konsens mit Mitgliedern des wissenschaftlichen Beirats des Netzwerks entwickelt. Diesem gehören Wissenschaftler verschiedener Fachrichtungen und Institutionen an (s. Autoren). Die Handlungsempfehlungen wenden sich an Schwangere in Deutschland und sollen die einheitliche Basis für Empfehlungen zur Ernährung in der Schwangerschaft für alle Berufsgruppen und Fachorganisationen sein, die werdende Eltern beraten.

Die Handlungsempfehlungen werden unterstützt von den Berufsverbänden und wissenschaftlichen Fachgesellschaften der Frauenärzte, der Hebammen sowie der Kinder- und Jugendärzte (Berufsverband der Frauenärzte, Deutscher Hebammenverband. Berufsverband der Kinder- und Jugendärzte, Deutsche Gesellschaft für Gynäkologie und Geburtshilfe, Deutsche Gesellschaft für Hebammenwissenschaft, Deutsche Gesellschaft für Kinder- und Jugendmedizin).

\section{Ergebnisse}

Im Folgenden wird eine Auswahl an Handlungsempfehlungen zu den in Fachkreisen oft diskutierten Themen Gewichtsentwicklung, Ernährungsweise, Supplemente, vegetarische Ernährung, vegane Ernährung sowie Bewegung vorgestellt und die Hintergründe dazu erläutert. 


\section{Gewichtsentwicklung}

\section{Empfehlungen}

- Das Körpergewicht der Frau vor der Schwangerschaft ist von großer Bedeutung für ihre Empfängnisbereitschaft, den Schwangerschafts- und Geburtsverlauf sowie für die Gesundheit des Kindes. Deshalb sollten Frauen schon vor der Schwangerschaft Normalgewicht anstreben.

- Eine normale Gewichtszunahme in der Schwangerschaft liegt für normalgewichtige Frauen zwischen etwa 10 und $16 \mathrm{~kg}$.

Das Gewicht, mit dem eine Frau in die Schwangerschaft geht, wirkt sich stärker auf die Gesundheit von Mutter und Kind aus als die Gewichtszunahme während der Schwangerschaft [1,2]. In Deutschland sind etwa 20\% der Frauen zwischen 20 und 39 Jahren übergewichtig und weitere etwa $9-14 \%$ adipös [3]. Übergewichtige und adipöse Frauen haben in der Schwangerschaft ein erhöhtes Risiko für Gestationsdiabetes, Bluthochdruck, Frühgeburten und Geburtskomplikationen $[4,5]$. Übergewicht der werdenden Mutter erhöht ferner das Risiko für Übergewicht beim Kind und für die damit assoziierten Folgeerkrankungen sowie für Spina bifida, Herzfehler und multiple Fehlbildungen $[5,6]$. Frauen mit Übergewicht, aber auch Frauen mit Untergewicht, sollten schon vor einer Schwangerschaft Normalgewicht anstreben.

Ab dem 2. Schwangerschaftstrimenon tritt eine deutliche Gewichtszunahme ein, die sich zusammensetzt aus dem Gewicht von Fetus, Plazenta und Fruchtwasser sowie der Zunahme mütterlicher Gewebe wie Brust und Uterus, der Zunahme der Blutmenge, der extrazellulären Flüssigkeit sowie der Fettdepots. Eine normale Gewichtszunahme in der Schwangerschaft liegt nach den Empfehlungen der Weltgesundheitsorganisation bei $10-14 \mathrm{~kg}$ [7] und nach Goldberg bei $10-16 \mathrm{~kg}$ [8].
Das amerikanische Institute of Medicine (IOM) empfiehlt unterschiedliche Bereiche der Gewichtszunahme während der Schwangerschaft, je nach vorher bestehendem BMI: Unter- und Normalgewichtige sollen demnach mehr zunehmen als übergewichtige oder adipöse Frauen [9]. Für normalgewichtige Frauen empfiehlt das IOM eine Gewichtszunahme von 11,5-16 kg. Neuere Studienergebnisse stellen die generelle Anwendung dieser Empfehlungen, die auf Beobachtungsstudien in den USA beruhen, jedoch infrage, v.a. die für übergewichtige und adipöse Frauen [1]. So zeigen Daten der bundesweiten KiGGS-Studie, dass das Risiko für späteres kindliches Übergewicht zwar erhöht ist, wenn normalgewichtige Frauen in der Schwangerschaft viel zunehmen, allerdings ist die Effektgröße begrenzt: Mit einem Kilogramm zusätzlicher Gewichtszunahme steigt das spätere kindliche Übergewichtsrisiko nur um 1\% [10]. Bei übergewichtigen und adipösen Schwangeren in Bayern war eine den amerikanischen Empfehlungen entsprechende Gewichtszunahme zwar mit weniger Präeklampsie und nicht elektiven Kaiserschnitten, aber einer höheren Zahl von Diabetes, Frühgeburten, niedrigem Geburtsgewicht sowie erhöhter perinataler Mortalität verbunden [11]. Eine weitere retrospektive Beobachtungsstudie zeigte bei stark adipösen Schwangeren $\left(\mathrm{BMI}>40 \mathrm{~kg} / \mathrm{m}^{2}\right)$ ein vermindertes Komplikationsrisiko bei Gewichtsabnahme [4]. Diese Studienergebnisse unterstreichen, dass die Datenlage insgesamt nicht hinreichend ist, um die IOM-Empfehlungen als „Soll-Empfehlung“ für Deutschland zu übernehmen. Belastbare Empfehlungen für den wünschenswerten Gewichtsverlauf in der Schwangerschaft könnten sich zukünftig aus derzeit laufenden Interventionsstudien ergeben [12]. Zunächst sollten Übergewichtige und adipöse Schwangere in der Beratung besonders auf eine ausgewogene Ernährung und regelmäßige Bewegung hingewiesen werden.

\section{Ernährungsweise in der Schwangerschaft}

Empfehlungen

- Eine ausgewogene und abwechslungsreiche Ernährung ist für die Gesundheit der Schwangeren und für die Entwicklung des Kindes von großer Bedeutung.

- Die Ernährung in der Schwangerschaft orientiert sich an den allgemeinen Empfehlungen für eine ausgewogene Ernährung.

- Regelmäßige Mahlzeiten sind in der Schwangerschaft wünschenswert und förderlich für das Wohlbefinden der werdenden Mutter.

- Schwangere sollten auf einen regelmäßigen Verzehr von Gemüse, Obst, Vollkornprodukten, fettarmer Milch und fettarmen Milchprodukten, fettarmem Fleisch und (fettreichem) Meeresfisch achten. Zur Deckung des Bedarfs an Folat und Jod werden zusätzlich Supplemente empfohlen.

- In einer ausgewogenen Ernährung sind die Lebensmittelgruppen unterschiedlich gewichtet:

- Reichlich sollte es kalorienfreie/kalorienarme Getränke und pflanzliche Lebensmittel geben.

- Mäßig sollten tierische Lebensmittel gegessen werden, dabei bevorzugt fettarme Milch(-produkte), fettarmes Fleisch, fettarme Fleischwaren und fettreiche Meeresfische.

- Sparsam sollten Fette mit hohem Anteil gesättigter Fettsäuren sowie Süßigkeiten und Snackprodukte verzehrt werden.

Eine ausgewogene Ernährung und regelmäßige körperliche Bewegung vor und während der Schwangerschaft wirken sich nicht nur kurzfristig auf Mutter und Kind aus, sondern haben auch langfristig positive Auswirkungen auf Gesundheit und Wohlbefinden [19]. Der Mehrbedarf für zahlreiche Nährstoffe in der Schwangerschaft kann - mit Ausnahme von Folat und Jod - durch eine geeignete Lebensmittelauswahl gedeckt werden; der Verzehr von speziellen (diätetischen) Lebensmitteln ist 
im Allgemeinen nicht notwendig. Die Einnahme von Supplementen kann eine ausgewogene Ernährungsweise nicht ersetzen. Regelmäßige, über den Tag verteilte Mahlzeiten fördern das Wohlbefinden, wobei die Anzahl der Mahlzeiten pro Tag (ob beispielsweise 3 oder 5) von individuellen Bedürfnissen der Schwangeren abhängt.

Die Gewichtung der Lebensmittelgruppen in den Empfehlungen in „reichlich“, „mäßig“ und „sparsam“ (s. Abb. 1 aidErnährungspyramide) soll verdeutlichen, dass die verschiedenen Lebensmittelgruppen in unterschiedlichen Mengen zu einer bedarfsgerechten Nährstoff- und Energieversorgung beitragen.

\section{Der Flüssigkeitsbedarf steigt in der} Schwangerschaft proportional zum Energiebedarf nur leicht an - um etwa $300 \mathrm{ml}$ pro Tag in den letzten Monaten der Schwangerschaft [14]. Als Richtwert für die Gesamtzufuhr von Wasser aus Speisen und Getränken wird für 19- bis 50-Jährige 35 ml/kg Körpergewicht und Tag empfohlen; dieser Richtwert gilt auch für Schwangere [15]. Der sich daraus ergebende Richtwert für die tägliche Trinkmenge von etwa 1,5 Litern [15] sollte nicht unterschritten werden. Bei heißen Umgebungstemperaturen oder stärkerer körperlicher Betätigung ist eine höhere Trinkmenge notwendig.

Um dem zum Teil stark gestiegenen Bedarf an Vitaminen und Mineralstoffen gerecht zu werden, sollten bevorzugt nährstoffdichte Lebensmittel verzehrt werden (z.B. Gemüse, Obst, Vollkornprodukte, fettarme Milch[-produkte]). Der Verzehr von täglich 5 Portionen Gemüse und Obst ist wünschenswert [16]. Getreideprodukte, besonders aus Vollkorngetreide, und Kartoffeln enthalten einen hohen Anteil an Vitaminen, Mineralstoffen sowie Ballaststoffen und sollten daher auch in der Schwangerschaft reichlich verzehrt werden. Bei Kartoffeln und Getreide(-produkten) sollten fettarme Zubereitungen bevorzugt werden.

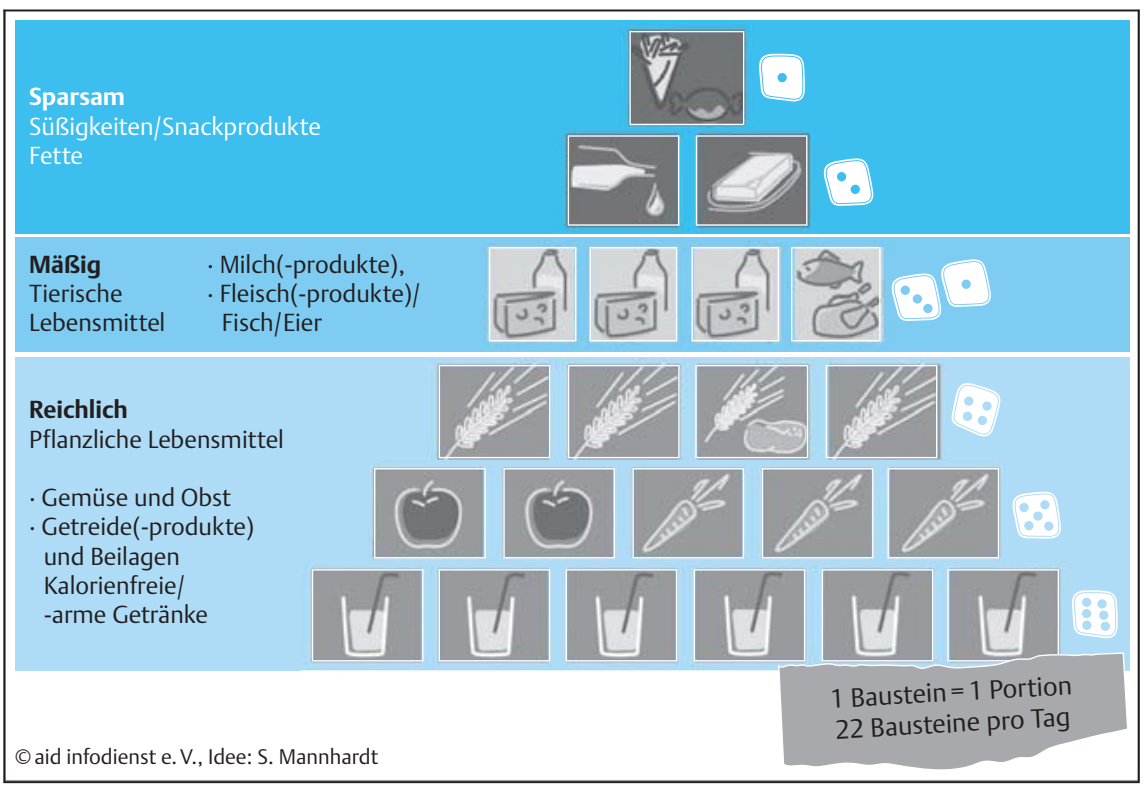

Abb. 1 aid-Ernährungspyramide. In einer ausgewogenen Ernährung sind die Lebensmittelgruppen unterschiedlich gewichtet. Jeder Baustein der Pyramide steht für eine Portion und das Maß für eine Portion ist die eigene Hand.

Fettarme Milch und Milchprodukte liefern Eiweiß, Kalzium, Jod und andere Nährstoffe und sind somit wichtige Bestandteile einer ausgewogenen Ernährung. Auch Fleisch als bedeutender Eisenlieferant sollte in der Ernährung der Schwangeren nicht fehlen. Eine Bevorzugung bestimmter eisenreicherer Fleischarten ist in einer gemischten Ernährung nicht erforderlich. Bei der Auswahl sollten v. a. fettarme Fleisch- und Wurstwaren bevorzugt werden. Süßigkeiten und Snackprodukte mit hoher Energie-, aber geringer Nährstoffdichte sollten nur in kleinen Mengen verzehrt werden.

Um die empfohlene Zufuhrmenge an langkettigen Omega-3-Fettsäuren von durchschnittlich mindestens $200 \mathrm{mg}$ Docosahexaensäure (DHA) pro Tag in der Schwangerschaft zu erreichen, sollten wöchentlich 2 Portionen Meeresfisch verzehrt werden, davon mindestens eine Portion fettreicher Meeresfisch (wie Makrele, Hering, Sardine, Lachs) [3,17]. Ein hoher Verzehr an Raubfischarten (z.B. Thunfisch, Schwertfisch), die am Ende der maritimen Nahrungskette stehen und hohe Schadstoffgehalte aufweisen können, sollte aus Gründen des vorbeugenden
Gesundheitsschutzes vermieden werden [18]. Schwangeren Frauen, die nicht regelmäßig Fisch verzehren, wird die Verwendung von Supplementen mit der Omega-3-Fettsäure DHA empfohlen [19]. Pflanzliche Öle sollten wegen ihres hohen Gehalts an einfach ungesättigten und essenziellen, mehrfach ungesättigten Fettsäuren regelmäßig verwendet werden. Dagegen wird eine sparsame Verwendung von Fetten mit einem hohen Anteil an gesättigten Fettsäuren (in der Regel „feste“ Fette) empfohlen.

Meeresfische tragen auch zur Versorgung mit Jod und Vitamin D (v.a. in fettreichen Arten) bei. Die Vitamin-D-Versorgung Schwangerer wirkt sich direkt auf die kindliche Vitamin-D-Versorgung und die kindliche Gesundheit aus, insbesondere auch auf die kindliche Knochenmineralisation $[20,21,22]$. Vitamin D wird mit der Ernährung zugeführt und durch Sonnenbestrahlung in der Haut gebildet, sodass durch regelmäßigen Aufenthalt im Freien die Vitamin-D-Versorgung sichergestellt werden kann. Bei einem hellen Hauttyp ist es dazu in unseren Breiten in den Sommermonaten ausreichend, wenn Gesicht und Arme etwa 5-10 Minuten täglich in 
der Mittagszeit ohne Lichtschutz der

Sonne ausgesetzt werden. Sonnenbrände sollten in jedem Fall vermieden werden. Die Deutsche Gesellschaft für Ernährung empfiehlt für Schwangere bei fehlender Vitamin-D-Eigensynthese eine VitaminD-Aufnahme von $20 \mu \mathrm{g}$ (800 IU) täglich [15]. Die mittlere Aufnahmemenge von Vitamin D über die Nahrung liegt jedoch nur bei 2-4 $\mu$ g täglich [3]. Diese Zufuhrmenge reicht nicht aus, um bei geringer endogener Synthese von Vitamin D im Winter und im Sommer im Falle begrenzter Sonnenexposition die wünschenswerte Serumkonzentration an 25(OH)Vitamin D von mindestens $50 \mathrm{nmol} / \mathrm{l} \mathrm{zu}$ erreichen. Schwangere, die sich selten im Sonnenlicht aufhalten oder bei Sonnenexposition ihre Haut weitgehend bedecken bzw. Sonnencreme anwenden, und Frauen mit dunklem Hauttyp, sollten ein Supplement mit Vitamin D verwenden, um die wünschenswerte Vitamin-DSerumkonzentration zu erreichen [15].

\section{Supplemente: Folsäure}

\section{Empfehlungen}

- Frauen, die eine Schwangerschaft planen, sollten zusätzlich zu einer ausgewogenen Ernährung zur Deckung des Folatbedarfs ein Supplement einnehmen (400 $\mu \mathrm{g}$ Folsäure/Tag).

- Die Einnahme soll mindestens bis zum Ende des 1. Schwangerschaftsdrittels fortgesetzt werden.

Die Folatversorgung ist u.a. wichtig für Zellteilung und Wachstumsprozesse. Folatreiche pflanzliche Lebensmittel wie grünes Blattgemüse, Kohlsorten, Hülsenfrüchte, Vollkornprodukte, Tomaten oder Orangen können zur Versorgung beitragen. Die mittlere Folatzufuhr der deutschen Bevölkerung liegt jedoch deutlich unter dem Referenzwert der DGE, der von 86\% der Frauen nicht erreicht wird [3]. In der Schwangerschaft steigt der Referenzwert für die Folatzufuhr (berechnet als Folatäquivalente) um $83 \%$ auf $550 \mu \mathrm{g} /$ Tag [15]. Eine Supplementierung mit täg- lich mindestens $400 \mu \mathrm{g}$ Folsäure (allein oder in Kombination mit anderen Mikronährstoffen) zeigte in zahlreichen Studien und in einer Cochrane-Metaanalyse eine ausgeprägte Risikominderung für kindliche Fehlbildungen des Nervensystems bzw. Neuralrohrdefekte [24,25]. In einzelnen Studien wurde auch eine Risikominderung für weitere angeborene Fehlbildungen wie z. B. angeborene Herzfehler und Lippen-Kiefer-Gaumenspalten berichtet [24,26-29]; jedoch ergab sich in der zitierten Cochrane-Metaanalyse insgesamt kein signifikanter Effekt für die Prävention weiterer angeborener Fehlbildungen [24]. Da der Verschluss des Neuralrohrs 3-4 Wochen nach der Konzeption erfolgt $[24,30]$, ist es für einen optimalen präventiven Effekt erforderlich, mit der Folsäuresupplementierung bereits vor der Konzeption zu beginnen. Frauen, die eine Schwangerschaft planen oder nicht ausschließen können, sollten zusätzlich zu einer ausgewogenen Ernährung, mindestens 4 Wochen vor der Konzeption, täglich ein Supplement mit $400 \mu \mathrm{g}$ Folsäure einnehmen und damit mindestens bis zum Ende des 1. Schwangerschaftsdrittels fortfahren [31]. Wenn die Einnahme erst kurz vor oder sogar erst nach der Konzeption beginnt, sollten Supplemente mit mehr als $400 \mu$ g Folsäure verwendet werden, da diese rascher zu einer präventiv wirksamen Gewebekonzentration führen [32]. Eine tägliche $\mathrm{Zu}-$ fuhr von bis zu $1000 \mu$ g Folsäure wird als sicher eingeschätzt [33].

Die Referenzwerte der DGE für die Zufuhr von Folatäquivalenten in der Schwangerschaft können allein mit der Nahrung praktisch nicht erreicht werden [23,34]. In Deutschland gibt es keine systematische Anreicherung von Grundnahrungsmitteln mit Folsäure [35]. Auch wenn einzelne Hersteller Lebensmittel mit Folsäure anreichern, wird Frauen vor und in der Schwangerschaft zur Einnahme von Folsäuresupplementen geraten. Derzeit führt nur ein geringer Teil der Frauen vor und in der Schwangerschaft eine präventiv wirksame Folsäuresupplementierung durch [36].
Auch über die 12. Schwangerschaftswoche hinaus kann die Einnahme eines Supplements mit Folsäure fortgesetzt werden. Zu möglichen Vorteilen von Multivitaminpräparaten mit Folsäure hinsichtlich der Risikominderung für Präeklampsie, der Verbesserung der oft schlechten Versorgung mit Vitamin D und ihrer Folgen für Mutter und Kind und der Senkung des Risikos für angeborene Fehlbildungen und für ein niedriges Geburtsgewicht des Kindes besteht weiterer Forschungsbedarf. Wenn Schwangere ein Multivitaminpräparat einnehmen, sollten sie darauf achten, dass es $400 \mu \mathrm{g}$ Folsäure enthält.

\section{Supplemente: Jod}

\section{Empfehlungen}

- Vor und in der Schwangerschaft sollte auf eine ausreichende Jodzufuhr geachtet werden. Empfehlenswert sind die Verwendung von jodiertem Speisesalz, der Verzehr von Meeresfisch 2-mal pro Woche sowie der regelmäßige Verzehr von Milch und Milchprodukten.

- Zusätzlich sollen Schwangere täglich ein Supplement mit $100(-150) \mu g$ Jod einnehmen. Bei Schilddrüsenerkrankungen soll eine Rücksprache mit dem behandelnden Arzt erfolgen.

Eine gute Jodversorgung ist nicht erst in der Schwangerschaft, sondern bereits vor der Befruchtung wichtig. Deshalb sollten Frauen mit Kinderwunsch zur Bedeutung von Jod beraten werden. Um eine gute Versorgung zu unterstützen, sollten im Haushalt und in der Gemeinschaftsverpflegung jodiertes Speisesalz eingesetzt und bei Lebensmitteln (z. B. Brot) bevorzugt Produkte ausgewählt werden, die mit jodiertem Speisesalz hergestellt wurden.

In der Schwangerschaft steigt der Referenzwert für die Jodzufuhr von 200 auf $230 \mu \mathrm{g}$ pro Tag an [15]. Durchschnittlich werden pro Tag etwa $120 \mu \mathrm{g}$ Jod mit der Nahrung und mit jodiertem Speisesalz aufgenommen. Da sich bereits eine milde Jodunterversorgung negativ auf die Ent- 
wicklung des Kindes auswirkt [37], sollen Schwangere zusätzlich zu einer ausreichenden Jodzufuhr durch die Nahrung täglich ein Supplement mit Jod einnehmen. Aufgrund der insgesamt verbesserten Jodversorgung erscheint ein Jodsupplement von 100-150 $\mu$ g pro Tag ausreichend. Dies entspricht dem unteren bis mittleren Bereich der in den Mutterschaftsrichtlinien genannten und als

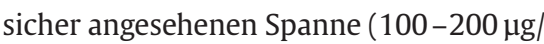
Tag) der Jodsupplementierung in der Schwangerschaft [38]. Werden andere Präparate eingenommen, die entsprechende Mengen an Jod enthalten, so sollten keine zusätzlichen Jodsupplemente eingenommen werden. Von der Verwendung getrockneter Algen- bzw. Tangpräparate mit möglichen exzessiv hohen Mengen an Jod wird abgeraten [39].

\section{Supplemente: Eisen}

\section{Empfehlungen}

- Schwangere sollten auf eine ausreichende Zufuhr von Eisen mit der Ernährung achten.

- Eine Eisensupplementierung ist individuell medizinisch abzuklären.

Der Eisenbedarf steigt in der Schwangerschaft an, weil mehr Eisen für Fetus, Plazenta und vermehrtes Blutvolumen der werdenden Mutter benötigt wird. Schwangere sollten auf eine ausreichende Zufuhr von Lebensmitteln achten, die gut resorbierbares Eisen enthalten. Dazu gehören Fleisch, Fleischwaren und Fisch. Auch pflanzliche Lebensmittel, wie Vollkornprodukte oder dunkle Gemüsearten, enthalten größere Mengen Eisen, das allerdings weniger gut bioverfügbar ist. Ein gleichzeitiger Verzehr von VitaminC-reichen Lebensmitteln (z. B. Zitrusfrüchte) kann die Eisenaufnahme verbessern.

Der Referenzwert für die Eisenzufuhr in der Schwangerschaft für Deutschland ist mit 30 mg/Tag um 100\% höher als der für Nichtschwangere [15]. Diese Zufuhrmenge wird in der Regel über die Nahrung nicht erreicht [23]. Allerdings entfällt der menstruelle Blutverlust, und die intestinale Eisenresorption in der Schwangerschaft steigt an [40]. Deshalb gehen einige Expertengruppen von einem etwa gleich hohen alimentären Eisenbedarf für Schwangere wie für Nichtschwangere aus $[40,41]$.

Eine generelle prophylaktische Eisensupplementierung wird Schwangeren nicht empfohlen, zumal eine erhöhte Eisenzufuhr bei Frauen, die gut mit Eisen versorgt sind, auch nachteilige Wirkungen haben kann [40,42]. Eine Supplementierung von Eisen sollte in der Schwangerschaft immer nur individuell und auf der Grundlage einer medizinischen Beratung erfolgen, die auf einer Anamnese und Blutuntersuchungen beruht.

\section{Vegetarische Ernährung in der Schwangerschaft}

\section{Empfehlungen}

- Eine vegetarische Ernährung mit Verzehr von Milch(-produkten) und Eiern (ovo-lakto-vegetarisch) kann bei gezielter Lebensmittelauswahl auch in der Schwangerschaft den Bedarf an den meisten Nährstoffen decken.

- Um eine ausreichende Eisenversorgung sicherzustellen, sollen nach entsprechender Blutuntersuchung und medizinischer Beratung ggf. Eisensupplemente eingesetzt werden.

- Bei Verzicht auf den Verzehr von Meeresfisch sollten Supplemente mit langkettigen Omega-3-Fettsäuren verwendet werden.

Mit einer ovo-lakto-vegetarischen Ernährung, bei der Lebensmittel gezielt ausgewählt und kombiniert werden, ist eine insgesamt gute Nährstoffversorgung in der Schwangerschaft möglich, mit Ausnahme von Vitamin D und der generell zu supplementierenden Nährstoffe Folsäure und Jod sowie - je nach ärztlich festgestelltem Eisenstatus - ggf. auch Eisen. Schwangere Vegetarierinnen, die keinen
Fisch essen, sollten Supplemente mit der Omega-3-Fettsäure DHA einnehmen, da die Eigensynthese aus dem in einigen Pflanzenölen enthaltenen Vorläufer Alpha-Linolensäure gering ist und eine Bedarfsdeckung nicht sicherstellen kann $[43,44]$. Fettreduzierte Milch und Milchprodukte, Eier, Hülsenfrüchte und Getreideprodukte gewährleisten in der Regel eine ausreichende Eiweißzufuhr. Eier, Hülsenfrüchte, (Vollkorn-)Getreideprodukte und einige Gemüsearten können zur Eisenzufuhr beitragen. Das Risiko für eine unzureichende Versorgung mit Eisen ist jedoch für Ovo-lakto-Vegetarierinnen erhöht $[45,46]$. Schwangere Frauen, die sich bereits vor der Schwangerschaft über lange Zeit vegetarisch ernährt haben, zeigen auch ein erhöhtes Risiko für einen Mangel an Vitamin $\mathrm{B}_{12}$ und Zink [45, 47-49]. In der Beratung von schwangeren Vegetarierinnen sollte daher besonderes Augenmerk auf die Versorgung mit diesen Mikronährstoffen gelenkt und bei Bedarf zur Einnahme von Supplementen geraten werden.

\section{Vegane Ernährung in der Schwangerschaft}

Empfehlungen

- Mit einer rein pflanzlichen (veganen) Ernährung ist eine ausreichende Nährstoffversorgung in der Schwangerschaft auch bei sorgfältiger Lebensmittelauswahl nicht möglich. Eine vegane Ernährung birgt ernsthafte gesundheitliche Risiken - insbesondere für die Entwicklung des kindlichen Nervensystems.

- Bei einer veganen Ernährung sind immer eine spezielle medizinische Beratung und die Einnahme von Mikronährstoffsupplementen notwendig.

Mit einer rein pflanzlichen, sog. veganen Ernährung ist v.a. die Zufuhr von ausreichend Energie, Eiweiß, langkettigen Omega-3-Fettsäuren, Eisen, Kalzium, Jod, Zink, Vitamin $B_{2}$, Vitamin $B_{12}$ und Vitamin $\mathrm{D}$ in der Schwangerschaft kritisch: die Gesundheitsrisiken für das Kind und die 
werdende Mutter sind damit hoch. Eine nach mehrjähriger veganer Ernährung aufgetretene Vitamin- $\mathrm{B}_{12}$-Unterversorgung kann während der Schwangerschaft zu schwerer und dauerhafter Schädigung des kindlichen Nervensystems führen [47, 50,51]. Veganerinnen, die ihre Ernährungsweise trotz Hinweis auf diese Risiken vor und in der Schwangerschaft beibehalten, brauchen eine qualifizierte Ernährungsberatung. Sie benötigen Supplemente, um den Nährstoffbedarf von Mutter und Kind zu decken.

\section{Bewegung}

Empfehlung

- Körperliche Aktivität in der Schwangerschaft ist wünschenswert und dient der Gesundheit von Mutter und Kind.

- Schwangere sollten täglich in Bewegung sein (körperliche Aktivitäten im Alltag und/oder Sport).

- Sportliches Training sollte in der Schwangerschaft nur mit mäßiger Intensität ausgeübt werden. Das ist der Fall, wenn eine Unterhaltung während des Sporttreibens noch möglich ist (Talk-Test).

In der Schwangerschaft scheint körperliche Aktivität mit moderater Intensität (kraft- und ausdauererhaltend, nicht steigernd) für Mutter und Kind von Vorteil zu sein (z.B. Steigerung des Wohlbefindens der Schwangeren, geringeres Risiko für Gestationsdiabetes und Präeklampsie, Vorbeugung einer hohen Gewichtszunahme). Regelmäßige körperliche Aktivität mit moderater Intensität wird empfohlen, sofern keine medizinischen Kontraindikationen vorliegen [1,52-54].

Frauen sollten in der Schwangerschaft keine neuen Sportarten mit ungewohnten Bewegungsabläufen beginnen. Für Schwangere nicht geeignet sind Sportarten mit hohem Sturz- und Verletzungsrisiko, z.B. Mannschafts-, Kontakt- und Kampfsportarten oder Sportarten wie Gerätetauchen. Empfehlenswert sind Sport-

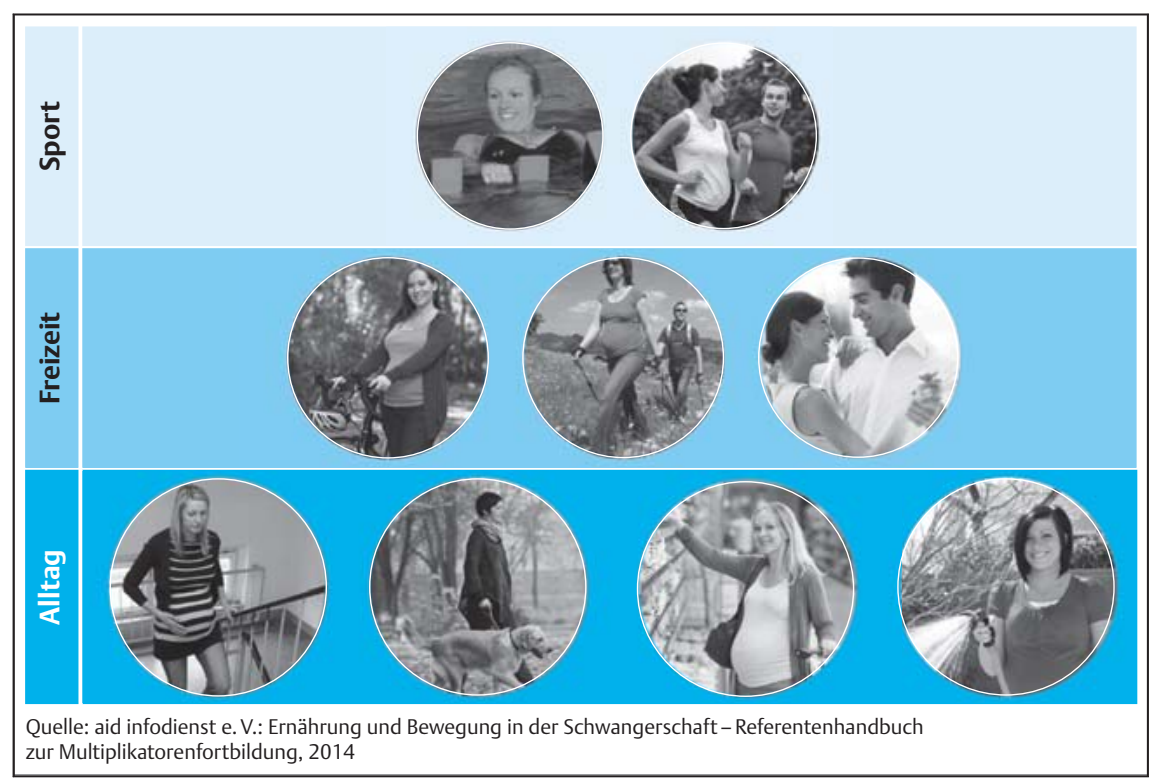

Abb. 2 Bewegungspyramide: Von Alltag bis Sport. Körperliche Aktivitäten können in 3 Arten unterteilt werden, die mit unterschiedlichen Intensitäten einhergehen: Alltags-, Freizeit- und sportliche Aktivitäten. Die Bewegungspyramide wurde in diesem Kontext für gesunde Schwangere konzipiert. Sie zeigt, wie diese 3 Aktivitäten gewichtet werden sollten:

Alltagsaktivitäten z. B. Wege zu Fuß oder mit dem Fahrrad erledigen, Treppensteigen, leichte Haus- und Gartenarbeiten bilden die Basis der Pyramide. Diese Aktivitäten machen einen großen Teil der Bewegungszeit aus. Freizeitaktivitäten wie Spaziergänge/Wandern, Fahrradfahren, Tanzen oder intensivere Gartenarbeit bilden das Mittelfeld der Pyramide. Diese Aktivitäten sind mäßig anstrengend, d. h., man kommt nur wenig außer Atem und/oder schwitzt.

Das obere Feld zeigt sportliche Aktivitäten wie Radfahren, Gymnastik, Schwimmen/Bewegung im Wasser oder Nordic Walking. Diese körperlichen Aktivitäten werden intensiv ausgeführt: Hier schwitzt man deutlich und die Atmung ist schwer.

Die Bilder in der Pyramide zeigen jeweils Beispiele für die jeweilige Art von Aktivität. Die aktuellen Bewegungsempfehlungen in der Schwangerschaft lauten: Schwangere sollten sich täglich bewegen: 30 Minuten am Tag an mindestens 5, möglichst allen Tagen in der Woche oder 150 Minuten pro Woche an moderater körperlicher Aktivität sind wünschenswert [64].

arten, die große Muskelgruppen beanspruchen, wie Radfahren, Schwimmen/ Bewegung im Wasser, aber auch Wandern oder Nordic Walking. Gesunde Schwangere können bis in Höhen von 2000-2500 m körperlich aktiv sein, v.a. wenn sie an diese Höhen gewöhnt sind $[55,56]$.

Regelmäßige Bewegung bzw. regelmäßiger Aufenthalt im Freien ist auch im Hinblick auf die Vitamin-D-Versorgung wünschenswert [38]. Schwangere Frauen weisen oft niedrige Vitamin-D-Konzentrationen im Blut auf, die mit schlechterer Knochenmineralisierung und Muskelmasse ihrer Kinder im Schulalter assoziiert sind [58-63]. Die durch Sonnenlicht induzierte Vitamin-D-Bildung in der Haut kann die Versorgung verbessern.

\section{Schlussfolgerungen}

Werdende Eltern sind im Hinblick auf den Nutzen für das Kind für gesundheitsfördernde Veränderungen ihres Lebensstils offen. Diese Chance sollten Ärzte, Ernährungsfachkräfte, Hebammen und weitere Multiplikatoren ergreifen und aktiv auf Paare mit Kinderwunsch und auf werdende Eltern zugehen, sie informieren und zu einer gesundheitsfördernden Ernährungsund Lebensweise motivieren. Diese im Konsens der hier relevanten Fachgesellschaften erarbeiteten Handlungsempfehlungen dienen als Grundlage für die Beratung zu Fragen der Ernährung und Bewegung in der Schwangerschaft. Dabei sollten Ärzte, Ernährungsfachkräfte, 


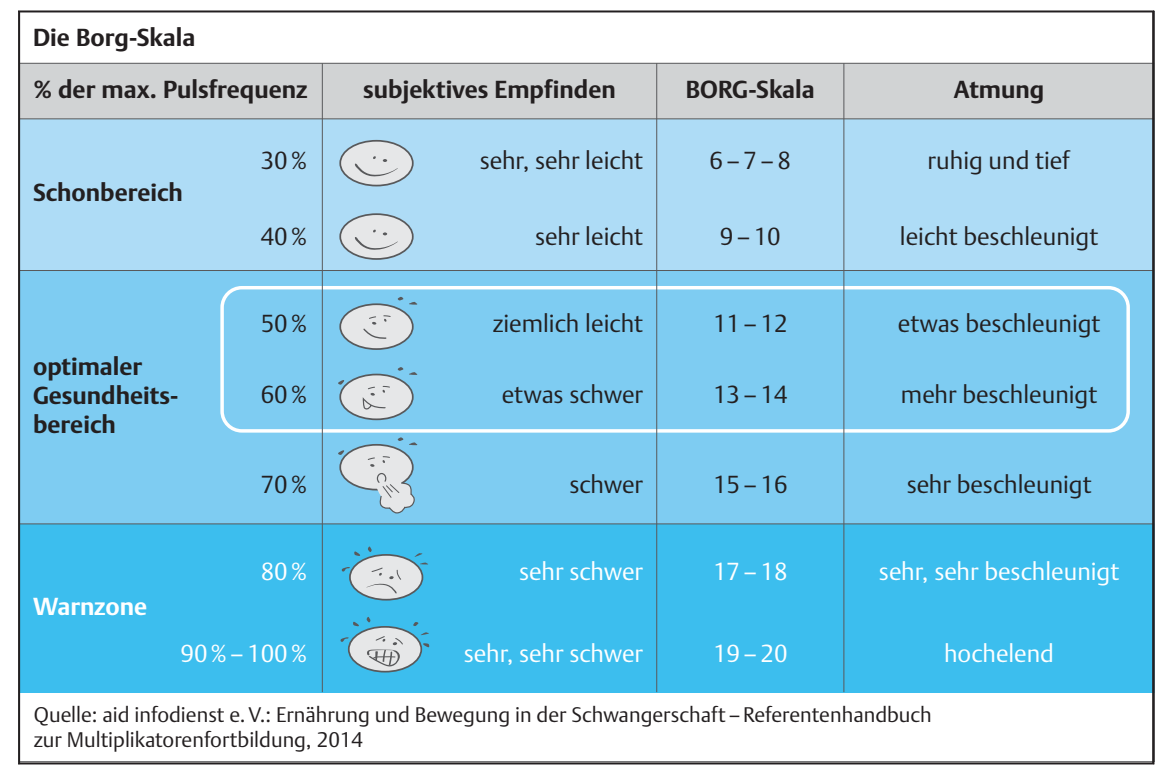

Abb. 3 Borg-Skala. Zur Bewertung des subjektiven Belastungsempfindens entwickelte der schwedische Physiologe Gunnar Borg die nach ihm benannte Borg-Skala, die im Laufe der Zeit modifiziert wurde. Die Skala stellt eine Einteilung nach verschiedenen Anstrengungsgraden von 6 (sehr, sehr leicht) bis 20 (sehr, sehr schwer) dar. Bei gesunden Schwangeren sollte das Belastungsempfinden zwischen 12 und 14, also im moderaten Bereich, liegen. Das bedeutet: leichtes Schwitzen und verstärkte Atmung [64].

Hebammen und weitere Multiplikatoren die Fragen und Sorgen der Zielgruppe ernst nehmen. Positive Botschaften sollten in der Beratung in den Vordergrund treten und die Freude auf das Kind unterstützt werden.

Schließlich geht es auch darum, die Handlungsempfehlungen bekannter zu machen. Deshalb bietet das Netzwerk zum einen zielgruppenspezifische Medien für Fachkräfte und junge Familien an. Zum anderen bilden die Handlungsempfehlungen die fachliche Grundlage für Multiplikatorenfortbildungen, die das Netzwerk „Gesund ins Leben“ bundesweit anbietet. Weitere Informationen unter http:// gesund-ins-leben.de/fuer-fachkraefte/ fortbildungen/schwangerschaft/ oder telefonisch 0228/8499-114.

\section{Weitere Informationen}

Das Netzwerk bietet für Fachkräfte und Familien Medien zum Thema Ernährung in der Schwangerschaft an. Weitere Informationen zu den Medien auf http:// gesund-ins-leben.de/fuer-fachkraefte/ medien-materialien/

\section{Interessenkonflikt}

Die Autoren geben keine Interessenkonflikte im Sinne des ICMJE (International Committee of Medical Journal Editors) an.

\section{Korrespondenzadresse}

M. Flothkötter

aid infodienst e. V.

Gesund ins Leben - Netzwerk Junge Familie Heilsbachstraße 16

53123 Bonn

Telefon: 0228/8499-129

Fax: 0228/8499-177

E-Mail: post@gesund-ins-leben.de

\section{Literatur}

1 National Institute for Health and Clinical Excellence. Dietary interventions and physical activity interventions for weight management before, during and after pregnancy. London: National Institute for Health and Clinical Excellence; 2010

2 Poston L. Intergenerational transmission of insulin resistance and type 2 diabetes. Prog Biophys Mol Biol 2011; 106: 315-322

3 Max Rubner Institut. Nationale Verzehrsstudie II, Ergebnisbericht. Karlsruhe: Bundesforschungsinstitut für Ernährung und Lebensmittel; 2008

4 Beyerlein A, Schiessl B, Lack $\mathrm{N}$ et al. Associations of gestational weight loss with birth-related outcome: a retrospective cohort study. BJOG 2011; 118: $55-61$

5 Cantwell R, Clutton-Brock T, Cooper G et al. Saving Mothers' Lives: Reviewing maternal deaths to make motherhood safer: 2006-2008. The Eighth Report of the Confidential Enquiries into Maternal Deaths in the United Kingdom. BJOG 2011; 118 (Suppl. 1): 1-203

6 EUROCAT. Special Report: a Review of Environmental Risk Factors for Congenital Anomalies. Belfast: EUROCAT Central Registry, University of Ulster; 2004

$7 \mathrm{FAO} / \mathrm{WHO} / \mathrm{UNU}$ Expert Consultation. Human energy requirements: report of a joint $\mathrm{FAO} / \mathrm{WHO} /$ UNU Expert Consultation. Food Nutr Bull 2005; 26: 166

8 Goldberg GR. Nutrition in pregnancy: the facts and fallacies. Nurs Stand 2003; 17: 39-42

9 Rasmussen KM, Yaktine AL eds. Committee to Reexamine IOM Pregnancy Weight Guidelines, Food and Nutrition Board and Board on Children, Youth, and Families; Institute of Medicine (US), National Research Council (US). Weight Gain During Pregnancy: Reexamining the Guidelines. Washington: 2009

10 Von Kries R, Ensenauer R, Beyerlein A et al. Gestational weight gain and overweight in children: result from the cross-sectional German KIGGS study. Int J Pediatr Obes 2011; 1: 45-52

11 Beyerlein A, Lack N, von Kries R. Within-population average ranges compared with Institute of Medicine recommendations for gestational weight gain. Obstet Gynecol 2010; 116 : 1111 1118

12 Scientific Advisory Committee on Nutrition. The influence of maternal, fetal and child nutrition on the development of chronic disease in later life. London: The Stationary Office; 2011

13 Koletzko B, Schiess S, Brands B et al. Frühkindliche Ernährung und späteres Adipositasrisiko. Hinweise auf frühe metabolische Programmierung. Bundesgesundheitsblatt, Gesundheitsforschung, Gesundheitsschutz 2010; 53: 666-673 
14 European Food Satety Authority Panel on Dietetic Products, Nutrition, and Allergies. Scientific opinion on dietary reference values for water. EFSA Journal 2010; 8: 1459-1507

15 Deutsche Gesellschaft für Ernährung, Österreichische Gesellschaft für Ernährung, Schweizerische Gesellschaft für Ernährung. Referenzwerte für die Nährstoffzufuhr. Bonn. 2. Auflage, 4. korrigierter Nachdruck ed. Frankfurt am Main: Umschau/Braus; 1. Ausgabe 2015

16 Deutsche Gesellschaft für Ernährung. Obst und Gemüse. Die Menge macht's. DGE info 2010; 1: 8-9

17 European Food Safety Authority Panel on Dietetic Products Nutrition and Allergies. Scientific opinion on dietary reference values for fats, including saturated fatty acids, polyunsaturated fatty acids, monounsaturated fatty acids, trans fatty acids, and cholesterol. EFSA Journal 2010; 8: 1461

18 European Food Safety Authority Panel on Contaminants in the food chain. Opinion of the Scientific Panel on Contaminants in the Food chain on a request from the Commission related to mercury and methylmercury in food. EFSA Journal 2004; 34: 1-14

19 Koletzko B, Cetin I, Brenna JT. Dietary fat intakes for pregnant and lactating women. Br J Nur 2007; 98: 873-877

20 Bischoff-Ferrari HA. Vitamin D: role in pregnancy and early childhood. Ann Nutr Metab 2011; 59 : $17-21$

21 Dawodu A, Wagner CL. Prevention of vitamin D deficiency in mothers and infants worldwide - a paradigm shift. Paediatr Int Child Health 2012; 32: $3-13$

22 Hyppönen E. Preventing vitamin D deficiency in pregnancy: importance for the mother and child. Ann Nutr Metab 2011; 2011

23 Max Rubner Institut. Nationale Verzehrsstudie II, Ergebnisbericht. Karlsruhe: Bundesforschungsinstitut für Ernährung und Lebensmittel; 2008

24 De-Regil LM, Fernandez-Gaxiola AC, Dowswell T et al. Effects and safety of periconceptional folate supplementation for preventing birth defects. Cochrane Database Syst Rev 2010; CD007950 10.1002/14651858.CD007950.pub2

25 Koletzko B, Pietrzik K. Gesundheitliche Bedeutung der Folsäurezufuhr. Dtsch Arztebl Int 2004; 101: A1670-A1681

26 Czeizel AE. Reduction of urinary tract and cardiovascular defects by periconceptional multivitamin supplementation. Am J Med Genet 1996; 62 : 179-183

27 Czeizel AE, Banhidy F. Vitamin supply in pregnancy for prevention of congenital birth defects. Current opinion in clinical nutrition and metabolic care 2011; 14: 291-296

28 Czeizel AE, Dudas I, Paput L et al. Prevention of neural-tube defects with periconceptional folic acid, methylfolate, or multivitamins? Ann Nutr Metab 2011; 58: 263-271

29 van Beynum IM, Kapusta L, Bakker MK et al. Protective effect of periconceptional folic acid supplements on the risk of congenital heart defects: a registry-based case-control study in the northern Netherlands. Eur Heart J 2010; 31: 464-471

30 Czeizel AE. Specified critical period of different congenital abnormalities: a new approach for human teratological studies. Congenit Anom (Kyoto) 2008; 48: 103-109

31 Becker S, Schmid D, Amann-Gassner U et al. Verwendung von Nährstoffsupplementen vor und während der Schwangerschaft. Ernährungs Umschau 2011; 58: 36-41

32 Bramswig S, Prinz-Langenohl R, Lamers Y et al. Supplementation with a multivitamin containing 800 microg of folic acid shortens the time to reach the preventive red blood cell folate concentration in healthy women. Int J Vitam Nutr Res 2009; 79: 61-70

33 Scientific Committee on Food, European Commission. Opinion of the Scientific Committee on Food on the tolerable upper intake level of folate. Brüssel: Europäische Kommission; 2000

34 Deutsche Gesellschaft für Ernährung, Deutsche Gesellschaft für Humangenetik, Deutsche Gesellschaft für Kinderheilkunde, Gesellschaft für Neuropädiatrie. Prävention von Neuralrohrdefekten durch Folsäurezufuhr in der Frühschwangerschaft. Der Frauenarzt 1994; 35: 1007-1010 Nachdruck in: Der Kinderarzt 1995; 26: 187-190; 2: ASbH-Brief - Zeitschrift der Arbeitsgemeinschaft Spina bifida und Hydrocephalus e. V.; 3: 1995; 1995. Monatsschr Kinderheilkd 1995; 143 : 1003-1005

35 Herrmann W, Obeid R. The mandatory fortification of staple foods with folic acid: a current controversy in Germany. Deutsches Arzteblatt international 2011; 108: 249-254

36 Inskip HM, Crozier SR, Godfrey KM et al. Women's compliance with nutrition and lifestyle recommendations before pregnancy: general population cohort study. BMJ 2009; 338: b481

37 Remer T, Johner SA, Gartner R et al. Jodmangel im Säuglingsalter - ein Risiko für die kognitive Entwicklung. Dtsch Med Wochenschr 2010; 135 : $1551-1556$

38 Bundesausschuss der Ärzte und Krankenkassen. Richtlinien des Bundesausschusses der Ärzte und Krankenkassen über die ärztliche Betreuung während der Schwangerschaft und nach der Entbindung („Mutterschafts-Richtlinien“). Bundesanzeiger 2010; 75: 1784

39 Bundesinstitut für Risikobewertung. Gesundheitliche Risiken durch zu hohen Jodgehalt in getrockneten Algen. Aktualisierte Stellungnahme Nr. 026/2007. Berlin: Bundesinstitut für Risikobewertung; 2007
40 Scientific Advisory Committee on Nutrition. Iron and Health. London: The Stationery Office; 2010

41 World Health Organization. Vitamin and mineral requirements in human nutrition. 2 nd ed. Geneva: World Health Organization and Food and Agriculture Organization of the United Nations; 2004

42 Pena-Rosas JP, Viteri FE. Effects and safety of preventive oral iron or iron+folic acid supplementation for women during pregnancy. Cochrane Database Syst Rev 2009; CD004736

43 Glaser C, Lattka E, Rzehak P et al. Genetic variation in polyunsaturated fatty acid metabolism and its potential relevance for human development and health. Matern Child Nutr 2011; 7 (Suppl. 2): 27-40

44 Lattka E, Klopp N, Demmelmair H et al. Genetic variations in polyunsaturated fatty acid metabolism - implications for child health? Ann Nutr Metab 2012; 60: 8-17

45 Wada L, King JC. Trace element nutrition during pregnancy. Clin Obstet Gynecol 1994; 37: 574 586

46 Wagener IE, Bergmann RL, Kamtsiuris P et al. Prävalenz und Risikofaktoren für Eisenmangel bei jungen Müttern. Gesundheitswesen 2000; 62: 176-178

47 Dror DK, Allen LH. Effect of vitamin B12 deficiency on neurodevelopment in infants: current knowledge and possible mechanisms. Nutr Rev 2008; 66: 250-255

48 King JC, Stein T, Doyle M. Effect of vegetarianism on the zinc status of pregnant women. Am J Clin Nutr 1981; 34: 1049-1055

49 Koebnick C, Hoffmann I, Dagnelie PC et al. Longterm ovo-lacto vegetarian diet impairs vitamin B-12 status in pregnant women. J Nutr 2004; 134: 3319-3326

50 Honzik T, Adamovicova M, Smolka V et al. Clinical presentation and metabolic consequences in 40 breastfed infants with nutritional vitamin B12 deficiency - what have we learned? Eur J Paediatr Neurol 2010; 14: 488-495

51 von Schenck U, Bender-Gotze C, Koletzko B. Persistence of neurological damage induced by dietary vitamin B-12 deficiency in infancy. Arch Dis Child 1997; 77: 137-139

52 Artal R, O'Toole M. Guidelines of the American College of Obstetricians and Gynecologists for exercise during pregnancy and the postpartum period. Br J Sports Med 2003; 37: 6-12; discussion 12

53 Committee on Obstetric Practice. ACOG committee opinion. Exercise during pregnancy and the postpartum period. Number 267, January 2002. American College of Obstetricians and Gynecologists. Int J Gynaecol Obstet 2002; 77 : 79-81 
54 National Institute for Health and Clinical Excellence. Dietary interventions and physical activity interventions for weight management before, during and after pregnancy. London: National Institute for Health and Clinical Excellence; 2010

55 Baumann H, Bung P, Fallenstein F et al. Reaction of mother and fetus to physical stress at high altitude. Geburtshilfe Frauenheilkd 1985; 45: 869876

56 Huch R. Physical activity at altitude in pregnancy. Semin Perinatol 1996; 20: 303-314

57 Böhles HJ, Fusch C, Genzel-Boroviczény O et al. Vitamin-D-Versorgung im Säuglings-, Kindesund Jugendalter. Stellungnahme der Ernährungskommission der Deutschen Gesellschaft für Kinder- und Jugendmedizin. Monatsschrift Kinderheilkunde 2011; 159: 766-774
58 Brannon PM, Picciano MF. Vitamin D in pregnancy and lactation in humans. Annu Rev Nutr 2011; 31: $89-115$

59 Gale CR, Robinson SM, Harvey NC et al. Maternal vitamin D status during pregnancy and child outcomes. Eur J Clin Nutr 2008; 62: 68-77

60 Hyppönen E. Preventing vitamin D deficiency in pregnancy: importance for the mother and child. Ann Nutr Metab 2011; 2011

61 Javaid MK, Crozier SR, Harvey NC et al. Maternal vitamin D status during pregnancy and childhood bone mass at age 9 years: a longitudinal study. Lancet 2006; 367: 36-43

62 Krishnaveni GV, Veena SR, Winder NR et al. Maternal vitamin D status during pregnancy and body composition and cardiovascular risk markers in Indian children: the Mysore Parthenon Study. Am J Clin Nutr 2011; 93: 628-635
63 Scientific Advisory Committee on Nutrition. The influence of maternal, fetal and child nutrition on the development of chronic disease in later life. London: The Stationary Office; 2011

64 aid infodienst e. V. Ernährung und Bewegung in der Schwangerschaft - Referentenhandbuch zur Multiplikatorenfortbildung. 2014; 127-131

Dieser Artikel ist eine Kurzfassung des zweiteiligen Beitrags „Ernährung in der Schwangerschaft“ in: Dtsch med Wochenschr 2012; 137: 1366-1372 und 137: 1309-1314 